\title{
OSCILLATION CRITERIA FOR SECOND-ORDER NONLINEAR SELF-ADJOINT DIFFERENTIAL EQUATIONS*
}

\author{
M. HESAARAKI ${ }^{\dagger}$ AND A. MORADIFAM ${ }^{\ddagger}$
}

Dedicated to Professor Joel Smoller on the occasion of his 70th birthday

Abstract. Our concern is to solve the oscillation problem for the nonlinear self-adjoint equation $\left(a(t) x^{\prime}\right)^{\prime}+b(t) g(x)=0$, where $g(x)$ satisfies the Signum condition $x g(x)>0$ if $x \neq 0$, but is not imposed such monotonicity as superlinear or sublinear. The problem has not been solved for the critical cases:

$$
\liminf _{|x| \rightarrow 0} \frac{g(x)}{x}<\frac{1}{4}<\limsup _{|x| \rightarrow 0} \frac{g(x)}{x}, \text { and } \liminf _{|x| \rightarrow \infty} \frac{g(x)}{x}<\frac{1}{4}<\limsup _{|x| \rightarrow \infty} \frac{g(x)}{x},
$$

which are more difficult, by now. We concentrate our attention on this point and give some answers. Sufficient conditions are given for all nontrivial solutions to be oscillatory.

Key words. Oscillation, nonlinear self-adjoint differential equations, Liénard-type system.

AMS subject classifications. Primary 34C10, 34C15; Secondary 34A12

1. Introduction. In this paper, we are concerned to obtain some oscillation criteria for the nonlinear self-adjoint differential equation

$$
\left(a(t) x^{\prime}\right)^{\prime}+b(t) g(x)=0,
$$

where $a(t)$ and $b(t)$ are positive, continuous, and locally of bounded variation on some half-line $[\alpha, \infty)$, and $g(x)$ is continuous on $\mathbf{R}$ and

$$
x g(x)>0, \quad \text { for } \quad x \neq 0 .
$$

We assume that uniqueness is guaranteed for the solutions of (1.1) to the initial value problem. In [21, Appendix] the authors have proved that all solutions of (1.1) are continuable in the future time. Hence, it is worth while to discuss whether solutions of (1.1) are oscillatory or not.

A nontrivial solution $x(t)$ of (1.1) is said to be oscillatory if there exists a sequence $t_{k}$ tending to infinity such that $x\left(t_{k}\right)=0$. Otherwise, the solution is said to be nonoscillatory. For brevity, Eq.(1.1) is called oscillatory (respectively nonoscillatory) in case all nontrivial solutions are oscillatory(respectively nonoscillatory). Equation (1.1) naturally includes the nonlinear equation

$$
x^{\prime \prime}+c(t) g(x)=0, \quad t>0,
$$

as a special case.

Over the past few decades, a grate deal of efforts has been made on the oscillation and nonoscillation of solutions of $(1.1)$ (or (1.3)). Those results can be found in [1$25]$ and the references cited therein. For example, there are many studies on the oscillation for the Emden-Fowler differential equation

$$
x^{\prime \prime}+a(t)|x|^{\gamma} \operatorname{sgn} x=0,
$$

\footnotetext{
*Received March 15, 2005; accepted for publication June 8, 2007.

$\dagger$ Department of Mathematics, Sharif University of Technology, P.O.Box 11365-9415, Tehran, Iran (hesaraki@sina.sharif.edu).

${ }^{\ddagger}$ Department of Mathematics, The University of British Columbia, Vancouver, BC, V6T 1Z2, Canada (a.moradi@math.ubc.ca).
} 
where $\gamma>0$, is a constant. This equation is called superlinear if $\gamma>1$ and sublinear if $0<\gamma<1$ (see $[5-18,23,24])$.

Hille [11] extended a classical result of Kneser [12] and stated the following theorem on the oscillation of the linear differential equation:

$$
x^{\prime \prime}+a(t) x=0
$$

Theorem A. Let

$$
\omega_{*}=\liminf _{t \rightarrow \infty} t^{2} a(t) \quad \text { and } \quad \omega^{*}=\limsup _{t \rightarrow \infty} t^{2} a(t) .
$$

Then Equation (1.5) is oscillatory if $\omega_{*}>1 / 4$, nonoscillatory if $\omega^{*}<1 / 4$, and no conclusion can be drawn if either $\omega_{*}$ or $\omega^{*}$ equals $1 / 4$.

In [21], Sugie et al. discussed the oscillation and nonoscillation problems for (1.1) and gave some nonoscillation theorems which are classified into two cases:

$$
\int_{\alpha}^{\infty} \frac{1}{a(\tau)} d \tau=\infty
$$

and

$$
\int_{\alpha}^{\infty} \frac{1}{a(\tau)} d \tau<\infty
$$

In order to state their theorems, in addition to (1.2) we must make the following assumption on $g(x)$ :

$$
\int_{0}^{x} g(\tau) d \tau \leq \frac{1}{2} x^{2} \quad \text { for } \quad x \in \mathbf{R} .
$$

Consider the following three sequences of functions for positive large values of $x$ :

$$
\begin{gathered}
l_{1}(x)=2 \log x \quad \text { and } \quad l_{n+1}(x)=\log \left(l_{n}(x)\right), \\
L_{1}(x)=1 \quad \text { and } \quad L_{n+1}(x)=L_{n}(x) l_{n}(x), \\
S_{n}(x)=\sum_{k=1}^{n} \frac{1}{L_{k}(x)^{2}}
\end{gathered}
$$

for $n \in N$. Their oscillation theorems are stated in the following.

Theorem B. Let (1.2), (1.6), and (1.8) hold. Suppose that $a(t)$ and $b(t)$ satisfy

$$
a(t) b(t)\left(\int_{\alpha}^{t} \frac{1}{a(\tau)} d \tau\right)^{2} \leq 1,
$$

for $t$ sufficiently large, and there exists an $n \in N$ such that

$$
\frac{g(x)}{x} \leq \frac{1}{4} S_{n}\left(x^{2}\right)
$$


for $x>0$ or $x<0$ and $|x|$ sufficiently large. Then Eq. (1.1) is nonoscillatory.

Theorem C. Let (1.2), (1.7), and (1.8) hold. Suppose that $a(t)$ and $b(t)$ satisfy

$$
a(t) b(t)\left(\int_{t}^{\infty} \frac{1}{a(\tau)} d \tau\right)^{2} \leq 1
$$

for $t$ sufficiently large, and there exists an $n \in N$ such that

$$
\frac{g(x)}{x} \leq \frac{1}{4} S_{n}\left(x^{2}\right)
$$

for $x>0$ or $x<0$ and $|x|$ sufficiently small. Then Eq.(1.1) is nonoscillatory.

Also Sugie and Yamaoka in [23] investigated the oscillation problem for (1.1) and gave the following theorems.

Theorem D. Let (1.2), (1.6) hold. Suppose that $a(t)$ and $b(t)$ satisfy

$$
a(t) b(t)\left(\int_{\alpha}^{t} \frac{1}{a(\tau)} d \tau\right)^{2} \geq 1,
$$

for $t$ sufficiently large, and there exists a $\lambda$ with $\lambda>1 / 4$ and $n \in N$ such that

$$
\frac{g(x)}{x} \geq \frac{1}{4} S_{n-1}\left(x^{2}\right)+\frac{\lambda}{\left\{l_{n}\left(x^{2}\right)\right\}^{2}},
$$

for $|x|$ sufficiently large. Then Eq.(1.1) is oscillatory.

Theorem E. Let (1.2), (1.7) hold. Suppose that $a(t)$ and $b(t)$ satisfy

$$
a(t) b(t)\left(\int_{t}^{\infty} \frac{1}{a(\tau)} d \tau\right)^{2} \geq 1
$$

for $t$ sufficiently small, and there exists a $\lambda$ with $\lambda>1 / 4$ and $n \in N$ satisfying (1.14) for $|x|$ sufficiently large. Then Eq. (1.1) is oscillatory.

Theorem F. Assume (1.2) and (1.6) hold. Suppose that $a(t)$ and $b(t)$ satisfy

$$
a(t) b(t)\left(\int_{\alpha}^{t} \frac{1}{a(\tau)} d \tau\right)^{2}=1
$$

and let

$$
h_{n}(x)=\left\{l_{n}(x)\right\}^{2}\left[\frac{g(x)}{x}-\frac{1}{4} S_{n-1}\left(x^{2}\right)\right] .
$$

Then Eq.(1.1) is oscillatory if

$$
\liminf _{|x| \rightarrow \infty} h_{n}(x)>\frac{1}{4}
$$

for some $n \in N$, and it is nonoscillatory if

$$
\limsup _{x \rightarrow \infty} h_{n}(x)<\frac{1}{4} \quad \text { or } \quad \limsup _{x \rightarrow-\infty} h_{n}(x)<\frac{1}{4},
$$


for some $n \in N$.

Theorem G. Assume (1.2) and (1.7) hold. Suppose that $a(t)$ and $b(t)$ satisfy

$$
a(t) b(t)\left(\int_{t}^{\infty} \frac{1}{a(\tau)} d \tau\right)^{2}=1
$$

Then Eq. (1.1) is oscillatory if

$$
\liminf _{|x| \rightarrow 0} h_{n}(x)>\frac{1}{4}
$$

for some $n \in N$, and it is nonoscillatory if

$$
\limsup _{x \rightarrow 0^{+}} h_{n}(x)<\frac{1}{4} \quad \text { or } \quad \limsup _{x \rightarrow 0^{-}} h_{n}(x)<\frac{1}{4},
$$

for some $n \in N$.

Notice that, all of the results presented in the above are inapplicable when

$$
\liminf _{|x| \rightarrow \infty} \frac{g(x)}{x}<\frac{1}{4}<\limsup _{|x| \rightarrow \infty} \frac{g(x)}{x} \quad \text { or } \quad \liminf _{|x| \rightarrow 0} \frac{g(x)}{x}<\frac{1}{4}<\limsup _{|x| \rightarrow 0} \frac{g(x)}{x} .
$$

In fact the oscillation problem has not been solved for this critical case which is more difficult, by now. In this paper we introduce some oscillation criteria for these critical cases.

2. Reduction to canonical forms. First consider the infinite case (1.6). Here by the following change of variable we transform our equation to a Liénard equation. The same idea is used in [23].

$$
s=s(t)=\int_{\alpha}^{t} \frac{1}{a(\tau)} d \tau
$$

Let $t(s)$ be the inverse function of $s(t)$ and put $v(s)=x(t(s))$. Then

$$
x^{\prime}(t)=\frac{d s}{d t} \dot{v}(s)=\frac{1}{a(t)} \dot{v}(s) \text { and }\left(a(t) x^{\prime}(t)\right)^{\prime}=\frac{d s}{d t} \ddot{v}(s)=\frac{1}{a(t)} \ddot{v}(s),
$$

and $v(s)$ satisfies the equation

$$
\ddot{v}+a(t(s)) b(t(s)) g(v)=0 .
$$

Let $c(s)=a(t(s)) b(t(s))$. Then condition (1.13) becomes

$$
t^{2} c(t) \geq 1
$$

for $t$ sufficiently large. Since $a(t)$ is positive for $t>\alpha$, the function $s(t)$ is increasing, and so is $t(s)$. From (1.6) it follows that $s(t)$ tends to infinity as $t \rightarrow \infty$ and $t(s)$ tends to infinity as $s \rightarrow \infty$. Hence, Eq.(1.1) is oscillatory if and only if the equation

$$
x^{\prime \prime}+c(t) g(x)=0,
$$


is oscillatory.

Now consider the finite case (1.7). Here we consider another change of variable for $t$ as follows

$$
s=s(t)=\left(\int_{t}^{\infty} \frac{1}{a(\tau)} d \tau\right)^{-1}
$$

Since $a(t)$ is positive, the function $s(t)$ and its inverse function $t(s)$ are increasing for $t>\alpha$ and $s>\beta$, respectively, where

$$
\beta=\left(\int_{\alpha}^{\infty} \frac{1}{a(\tau)} d \tau\right)^{-1}>0
$$

Since $a(t)$ satisfies (1.7), $s(t)$ tends to infinity as $t \rightarrow \infty$, and therefore, $t(s)$ also tends to infinity as $s \rightarrow \infty$. Let $v(s)=x(t(s))$. Then we have

$$
\begin{gathered}
x^{\prime}(t)=\frac{d s}{d t} \dot{v}(s)=\frac{s^{2}}{a(t)} \dot{v}(s) \\
\left(a(t) x^{\prime}(t)\right)^{\prime}=\frac{d s}{d t}\left(s^{2} \ddot{v}(s)+2 s \dot{v}(s)\right)=\frac{s^{4}}{a(t)}\left(\ddot{v}(s)+\frac{2}{s} \dot{v}(s)\right) .
\end{gathered}
$$

Put $c(s)=a(t(s)) b(t(s)) / s^{4}$. Then Equation (1.1) becomes

$$
x^{\prime \prime}+\frac{2}{t} x^{\prime}+c(t) g(x)=0, \quad \text { or } \quad\left(t^{2} x^{\prime}\right)^{\prime}+t^{2} c(t) g(x)=0, \quad t>\beta,
$$

and it is oscillatory if and only if (2.3) is oscillatory. Moreover in this case condition (1.15) coincides with the condition (2.1).

Let $s=\log t$ and $u(s)=x\left(e^{s}\right)=x(t)$. Then Eqs.(2.2) and (2.3) are transformed into the systems

$$
\dot{u}=v+u, \quad \dot{v}=-e^{2 s} c\left(e^{s}\right) g(u)
$$

and

$$
\dot{u}=v-u, \quad \dot{v}=-e^{2 s} c\left(e^{s}\right) g(u),
$$

respectively. The systems (2.4) and (2.5) are of Liénard type. Hereafter we denote $s$ by $t$ again.

Now consider the systems

$$
\dot{u}=v+u, \quad \dot{v}=-C(t) g(u),
$$

and

$$
\dot{u}=v-u \quad \dot{v}=-C(t) g(u),
$$

where $C(t) \geq 1$. Notice, Eqs. (2.4) and (2.5) are special cases of (2.6) and (2.7), respectively.

We say that the system $(2.6)$ (or $(2.7))$ has property $\left(X^{+}\right)$in the right half plane (resp. in the left half plane), if for every point $\left(x_{0}, y_{0}\right)$ with $y_{0}>-x_{0}$ (or $\left.y_{0}>x_{0}\right)$ and $x_{0} \geq 0$ (resp. $y_{0}<-x_{0}$ (or $\left.y_{0}<x_{0}\right)$ and $x_{0} \leq 0$ ), the positive semitrajectory of 
(2.6)(or 2.7) passing through $\left(x_{0}, y_{0}\right)$ crosses the vertical isocline $y=-x$ (or $y=x$ ) (see $[1,3])$.

Let

$$
G(x)=\int_{0}^{x} g(\eta) d \eta
$$

Theorem 2.1. The system (2.6) has property $\left(X^{+}\right)$in the right half plane if

$$
\limsup _{x \rightarrow+\infty}\left(\int_{b}^{x}\left(\frac{-\eta g(\eta)}{(2 G(\eta))^{\frac{3}{2}}}+\frac{g(\eta)}{G(\eta)}\right) d \eta-\frac{x}{\sqrt{2 G(x)}}\right)=+\infty
$$

for some $b>0$.

Proof. We prove the theorem by contradiction. Suppose that there exists a solution $(u(t), v(t))$ of $(2.6)$ whose graph remains in the region $\{(u, v): u \geq 0$ and $v>$ $-u\}$ for all future time. Let $\left(u_{t_{0}}, v_{t_{0}}\right)=(u(0), v(0))$. Since, the system $(2.6)$ has no critical points in this region, we have

$$
u(t) \rightarrow+\infty \quad \text { as } t \rightarrow+\infty,
$$

so, we may assume that $u_{0}>0$. Now let

$$
f(t)=\int_{b}^{u(t)} \frac{-\eta g(\eta)}{(2 G(\eta))^{\frac{3}{2}}} d \eta+\frac{v(t)}{\sqrt{2 G(u(t)})} .
$$

Then

$$
\begin{gathered}
\dot{f}(t)=\dot{u}(t) \frac{-u(t) g(u(t))}{\left(2 G(u(t)) \frac{3}{2}\right.}+\frac{2 \dot{v}(t) G(u(t))-v(t) t u(t) g(u(t))}{(2 G(u(t))) \frac{3}{2}} \\
=-\frac{g(u(t)) \dot{u}(t)(u(t)+v(t))-2 \dot{v}(t) G(u(t))}{(2 G(u(t))) \frac{3}{2}} \\
=-\frac{g(u(t)) \dot{u}^{2}(t)+2 g(u(t)) C(t) G(u(t))}{(2 G(u(t))) \frac{3}{2}} .
\end{gathered}
$$

Since, $C(t) \geq 1$, we have

$$
\dot{f}(t) \leq-\dot{u}(t) \frac{g(u(t))}{G(u(t))}
$$

Thus,

$$
\frac{d}{d t}\left(f(t)+\int_{b}^{u(t)} \frac{g(\eta)}{G(\eta)} d \eta\right) \leq 0 .
$$

Therefore,

$$
\int_{b}^{u(t)}\left(\frac{-\eta g(\eta)}{(2 G(\eta))^{\frac{3}{2}}}+\frac{g(\eta)}{G(\eta)}\right) d \eta+\frac{v(t)}{\sqrt{2 G(u(t)})} \leq
$$




$$
\int_{b}^{u_{0}}\left(\frac{-\eta g(\eta)}{(2 G(\eta))^{\frac{3}{2}}}+\frac{g(\eta)}{G(\eta)}\right) d \eta+\frac{v_{0}}{\sqrt{2 G\left(u_{0}\right)}}<+\infty
$$

for $t \geq t_{0}$. Since, $v(t)>-u(t)$ and $u(t) \rightarrow+\infty$ as $t \rightarrow+\infty$, thus,

$$
\limsup _{x \rightarrow+\infty}\left(\int_{b}^{x}\left(\frac{-\eta g(\eta)}{(2 G(\eta))^{\frac{3}{2}}}+\frac{g(\eta)}{G(\eta)}\right) d \eta-\frac{x}{\sqrt{2 G(x)}}\right)<+\infty .
$$

This contradiction completes the proof.

The following analogous result is obtained with respect to property $\left(X^{+}\right)$in the left half plane.

Theorem 2.2. The system (2.6) has property $\left(X^{+}\right)$in the left half plane if

$$
\liminf _{x \rightarrow-\infty}\left(\int_{x}^{b}\left(\frac{\eta g(\eta)}{(2 G(\eta))^{\frac{3}{2}}}+\frac{g(\eta)}{G(\eta)}\right) d \eta+\frac{x}{\sqrt{2 G(x)}}\right)=-\infty,
$$

for some $b<0$.

Lemma 2.1. For each point $C=(c,-c)$ with $c>0$, the positive semitrajectory of (2.6) passing through $C$ crosses the negative y-axis.

Proof. Suppose that there exists a point $C=(c,-c)$ with $c>0$ such that the positive semitrajectory of (2.6) passing through $C=(c,-c)$ does not intersect the negative $y$-axis. Let $(x(s), y(s))$ be a solution of $(2.6)$ defined on an interval $\left[s_{0}, \infty\right)$ with $\left(x\left(s_{0}\right), y\left(s_{0}\right)\right)=C$. Then we have

$$
0<x(s) \leq x\left(s_{0}\right) \text { for } s \geq s_{0}
$$

and

$$
y(s) \rightarrow-\infty \text { as } s \rightarrow \infty .
$$

Hence, it follows from the first equation of (2.6) that

$$
\dot{x}(s) \rightarrow-\infty \text { as } s \rightarrow \infty,
$$

and therefore, there exists an $s_{1}>s_{0}$ such that

$$
\dot{x}(s) \leq-1 \text { for } s \geq s_{1} .
$$

Integration of the above leads to

$$
-x\left(s_{1}\right)<x(s)-x\left(s_{1}\right) \leq s_{1}-s \rightarrow-\infty \text { as } s \rightarrow-\infty .
$$

This is a contradiction and the proof is complete.

Similarly, turning our attention to the left half plane we have the following result. 
Lemma 2.2. For each point $C=(-c, c)$ with $c>0$, the positive semitrajectory of (2.6) passing through $C$ crosses the positive y-axis.

Lemma 2.3. The system (2.7) has property $\left(X^{+}\right)$in the right and left half plane.

Proof. Let $x_{0}>0, y_{0}>x_{0}$, and $(x(t), y(t))$ be the solution of (2.7) passing through $\left(x_{0}, y_{0}\right)$. Since, $\dot{x}(t)>0$ and $\dot{y}(t)<0$ for $x(t)>0$ and $y(t)>x(t)$, this orbit must intersect the line $y=x$, otherwise there must be a rest point in the first quadrant, which is impossible. Similarly, we can conclude that the system (2.7) has property $\left(X^{+}\right)$in the left half plane.

Suppose that the system $(2.7)$ has a solution $u(t)$ starting at $(c, c)$ and does not intersect the negative $\mathrm{y}$-axis, we can show that $u(t)$ tends to zero as $t \rightarrow \infty$, (see [23, Lemma 5.1] for the proof). From $\dot{u}=v+u$, we conclude that $v(t) \rightarrow 0$, when $t \rightarrow \infty$. Since, $\dot{v}(t)<0, v(t)>0$, for all future time. Now we are ready to prove the following theorem.

Theorem 2.3. Suppose that

$$
\liminf _{x \rightarrow 0^{+}}\left(\int_{x}^{b}\left(\frac{\eta g(\eta)}{(2 G(\eta))^{\frac{3}{2}}}-\frac{g(\eta)}{G(\eta)}\right) d \eta\right)=-\infty, \quad \text { for some } b>0 .
$$

Then for each point $C=(c, c)$ with $c>0$, the positive semitrajectory of (2.7) passing through $C$ crosses the negative y-axis.

Proof. Suppose that there exists a point $C=(c, c)$ with $c>0$ such that the positive semitrajectory of (2.7) passing through $C=(c, c)$ does not intersect the negative $y$-axis.

Let

$$
f(t)=\int_{b}^{u(t)} \frac{\eta g(\eta)}{(2 G(\eta))^{\frac{3}{2}}} d \eta+\frac{v(t)}{\sqrt{2 G(u(t)})} .
$$

Then

$$
\begin{aligned}
\dot{f}(t)= & \dot{u}(t) \frac{u(t) g(u(t))}{(2 G(u(t))) \frac{3}{2}}+\frac{2 \dot{v}(t) G(u(t))-v(t) \dot{u}(t) g(u(t))}{(2 G(u(t))) \frac{3}{2}} \\
= & -\frac{g(u(t)) \dot{u}(t)(v(t)-u(t))-2 \dot{v}(t) G(u(t))}{(2 G(u(t))) \frac{3}{2}} \\
& =-\frac{g(u(t)) \dot{u}^{2}(t)+2 g(u(t)) C(t) G(u(t))}{(2 G(u(t))) \frac{3}{2}} .
\end{aligned}
$$

Since, $C(t) \geq 1$, we have

$$
\dot{f}(t) \leq \dot{u}(t) \frac{g(u(t))}{G(u(t))} .
$$


Thus,

$$
\frac{d}{d t}\left(f(t)-\int_{b}^{u(t)} \frac{g(\eta)}{G(\eta)} d \eta\right) \leq 0
$$

Therefore,

$$
\begin{gathered}
\int_{b}^{u(t)}\left(\frac{\eta g(\eta)}{(2 G(\eta))^{\frac{3}{2}}}-\frac{g(\eta)}{G(\eta)}\right) d \eta+\frac{v(t)}{\sqrt{2 G(u(t)})} \leq \\
\int_{b}^{c}\left(\frac{\eta g(\eta)}{(2 G(\eta))^{\frac{3}{2}}}-\frac{g(\eta)}{G(\eta)}\right) d \eta+\frac{c}{\sqrt{2 G(c)}}<\infty \quad \text { for } t \geq t_{0},
\end{gathered}
$$

where $\left(\left(u\left(t_{0}\right), v\left(t_{0}\right)\right)=(c, c)\right.$. Since, $u(t) \rightarrow 0^{+}$as $t \rightarrow+\infty$, and $v(t)>0$ we have

$$
\liminf _{x \rightarrow 0^{+}}\left(\int_{x}^{b}\left(\frac{\eta g(\eta)}{(2 G(\eta))^{\frac{3}{2}}}-\frac{g(\eta)}{G(\eta)}\right) d \eta\right)>-\infty .
$$

This contradiction completes the proof.

Turning our attention to the left half plane, similarly we have the following result.

Theorem 2.4. Suppose that

$$
\liminf _{x \rightarrow 0^{-}}\left(\int_{b}^{x}\left(\frac{\eta g(\eta)}{(2 G(\eta))^{\frac{3}{2}}}+\frac{g(\eta)}{G(\eta)}\right) d \eta\right)=-\infty, \quad \text { for some } b<0 .
$$

Then for each point $C=(-c,-c)$ with $c>0$, the positive semitrajectory of $(2.7)$ passing through $C$ crosses the positive y-axis.

3. Oscillation Theorems. In this section we will present our main results and give some examples to illustrate our results.

Theorem 3.1. Suppose that (1.6), (1.13), (2.9) and (2.10) hold, then all nontrivial solutions of (1.1) are oscillatory.

Proof. Since (1.6), (1.13), (2.9) and (2.10) hold, the system (2.4) has property $\left(X^{+}\right)$in the right and left half plane. Thus, it follows from Lemmas 2.1 and 2.2 that every solution of (2.4) keeps on rotating around the origin except the zero solution. Hence, all nontrivial solutions of (2.4) are oscillatory. Thus, all nontrivial solutions of (1.1) are oscillatory, too. $\square$ If

Theorem 3.2. Suppose that (1.6) and (1.13) hold and $G(x)$ is defined by (2.8).

$$
\liminf _{|x| \rightarrow \infty} \frac{G(x)}{x^{2}}>\frac{1}{8}
$$

then all nontrivial solutions of (1.1) are oscillatory. 
Proof. Suppose that (3.1) holds, then

$$
\alpha=\liminf _{|x| \rightarrow \infty}\left(1-\frac{x}{2 \sqrt{2 G(x)}}\right)>0 .
$$

Hence, for $x>R$ with sufficiently large $R>0$ we have

$$
\begin{gathered}
\int_{R}^{x}\left(\frac{-\eta g(\eta)}{(2 G(\eta))^{\frac{3}{2}}}+\frac{g(\eta)}{G(\eta)}\right) d \eta=\int_{R}^{x} \frac{g(\eta)}{G(\eta)}\left(1-\frac{\eta}{2 \sqrt{2 G(\eta)}}\right) d \eta \\
\geq \frac{\alpha}{2} \int_{R}^{x} \frac{g(\eta)}{G(\eta)} d \eta=\frac{\alpha}{2}(\ln G(x)-\ln G(R)) .
\end{gathered}
$$

Therefore,

$$
\lim _{x \rightarrow+\infty} \int_{R}^{x}\left(\frac{-\eta g(\eta)}{(2 G(\eta))^{\frac{3}{2}}}+\frac{g(\eta)}{G(\eta)}\right) d \eta=+\infty .
$$

Since $\frac{x}{\sqrt{2 G(x)}}$ is bounded, (2.9) holds. Similarly, we can conclude that (2.10) holds, too. Then Theorem 3.1 implies that all nontrivial solutions of (1.1) must be oscillatory.

The following analogous results are obtained with respect to the finite case (1.7).

Theorem 3.3. Suppose that (1.7), (1.15), (2.11) and (2.12) hold, then Eq.(1.1) is oscillatory.

If

Theorem 3.4. Suppose that (1.7) and (1.15) hold and $G(x)$ is defined by (2.8).

$$
\liminf _{|x| \rightarrow 0} \frac{G(x)}{x^{2}}>\frac{1}{8}
$$

then Eq.(1.1) is oscillatory.

Here we give some examples to illustrate our results. The first example is related to Theorem 3.2.

EXAmple 3.1. Consider the following equation

$$
t x^{\prime \prime}+x^{\prime}+\frac{m x+n x \sin (x)}{t(\log (t))^{2}}=0,
$$

where $m>\max \left\{|n|, \frac{1}{4}\right\}$. Let

$$
a(t)=t, \quad b(t)=\frac{1}{t(\log (t))^{2}},
$$

and

$$
g(x)=m x+n x \sin (x) .
$$


Obviously (1.6) holds and

$$
a(t) b(t)\left(\int_{1}^{t} \frac{1}{a(\tau)} d \tau\right)^{2}=1 .
$$

We have

$$
G(x)=\frac{m}{2} x^{2}-n x \cos (x)+n \sin (x)
$$

Thus,

$$
\liminf _{|x| \rightarrow \infty} \frac{G(x)}{x^{2}}=\frac{m}{2}>\frac{1}{8} .
$$

Therefore, by Theorem 3.2 Eq.(1.1) is oscillatory.

EXAMPLE 3.2. Consider the following equation

$$
\left(t^{\beta+2} x^{\prime}\right)^{\prime}+(\beta+1)^{2} t^{\beta} g(x)=0, \quad \beta>1 .
$$

Assume $g(0)=0$, and

$$
g(x)=m x+n x \sin \left(\frac{1}{x}\right), \quad \text { for } \quad x \neq 0,
$$

where $m>\max \left\{|n|, \frac{1}{4}\right\}$. Let

$$
a(t)=t^{\beta+2}, \quad \text { and } \quad b(t)=(\beta+1)^{2} t^{\beta},
$$

for $t \geq \alpha=1$. It is clear that $a(t)$ and $b(t)$ are positive and continuously differentiable for $t \geq 1$. We have

$$
\int_{1}^{t} \frac{1}{a(\tau)} d \tau<\frac{1}{\beta+1}<\infty, \quad a(t) b(t)\left(\int_{t}^{\infty} \frac{1}{a(\tau)} d \tau\right)^{2}=1
$$

for $t \geq 1$, and

$$
G(x)=\frac{m x^{2}}{2}+n \int_{0}^{x} \eta \sin \left(\frac{1}{\eta}\right) d \eta .
$$

Also

$$
\begin{gathered}
\lim _{x \rightarrow 0} \frac{\int_{0}^{x} \eta \sin \left(\frac{1}{\eta}\right) d \eta}{x^{2}}=\lim _{u \rightarrow \infty} \frac{\int_{\frac{1}{u}}^{\infty} \frac{\sin (\eta)}{\eta^{3}} d \eta}{\frac{1}{u^{2}}} \\
=\lim _{u \rightarrow \infty} \frac{\frac{-1}{u^{2}} \frac{\sin (u)}{u^{3}}}{\frac{-2}{u^{3}}}=0
\end{gathered}
$$

Thus,

$$
\lim _{|x| \rightarrow 0} \frac{G(x)}{x^{2}}=\frac{m}{2}>\frac{1}{8} .
$$


Therefore, by Theorem 3.4 Eq. (1.1) is oscillatory.

EXAmple 3.3. Consider the following equation

$$
t x^{\prime \prime}+x^{\prime}+\frac{\mu\left(x \exp \left(-x^{2}\right)+\alpha x \sin ^{2}(x)\right)}{t(\log (t+1))^{2}}=0 .
$$

Let

$$
a(t)=t, \quad \text { and } \quad b(t)=\frac{\mu}{t(\log (t+1))^{2}},
$$

and

$$
g(x)=x \exp \left(-x^{2}\right)+\alpha x \sin ^{2}(x) .
$$

We have

$$
a(t) b(t)\left(\int_{1}^{t} \frac{1}{a(\tau)} d \tau\right)^{2}=\mu\left(\frac{\log t}{\log (t+1)}\right)^{2} \nearrow \mu
$$

as $t \rightarrow \infty$. Hence, if $\mu>1$, the condition (1.13) is satisfied for $t$ sufficiently large. Also

$$
G(x)=\frac{\alpha}{4} x^{2}-\frac{1}{2} \exp \left(-x^{2}\right)-\frac{1}{8}(\alpha \cos (2 x)+2 \alpha x \sin (2 x)) .
$$

Thus,

$$
\liminf _{|x| \rightarrow \infty} \frac{G(x)}{x^{2}}=\frac{\alpha}{4}>\frac{1}{8} \quad \text { if } \quad \alpha>1 / 2 .
$$

Then Theorem 3.2 implies that all nontrivial solutions of (1.1) are oscillatory.

Notice that in Example 3.3

$$
\liminf _{|x| \rightarrow \infty} \frac{g(x)}{x}=0 .
$$

However, we have shown that Eq. (1.1) is oscillatory.

REMARK 3.1. Here we should mention that all the results presented in the previous literature are inapplicable to Examples 3.1, 3.2, and 3.3.

\section{REFERENCES}

[1] A. Aghajani, A. Moradifam, Some sufficient conditions for the intersection with the vertical isocline in the Liénard plane, Appl. Math. Lett., 19 (2006), pp. 491-497.

[2] A. Aghajani, A. Moradifam, Oscillation of solutions of second-order nonlinear differential equations of Euler type, J. Math. Anal. Appl., 326 (2007), pp. 1076-1089.

[3] A. Aghajani, A. Moradifam, Intersection with the vertical isocline in the Liénard plane, Nonlinear Analysis (2007), doi:10.1016/j.na.2007.03.040.

[4] R. Blaśko, J.R. Graef, M. HaĉIK, P.W. Spikes, Oscillatory behavior of solutions of nonlinear differential equations of the second order, J. Math. Anal. Appl., 151 (1990), pp. 330343.

[5] G.J. Butler, L.H. Erbe, A generalization of Olech-Opial-Ważewski oscillation criteria to second order nonlinear equations, Nonlinear Anal., 11 (1987), pp. 207-219. 
[6] M. Cecchi, M. Marini, G. Villari, On some classes of continuable solutions of a nonlinear differential equation, J. Differential Equations, 118 (1995), pp. 403-419.

[7] M. CECCHI, M. MARINI, G. VillaRi, Comparison results for oscillation of nonlinear differential equations, NoDEA Nonlinear Differential Equations Appl., 6 (1999), pp. 173-190.

[8] C.V. Coffman, J.S.W. Wong, Oscillation and nonoscillation of solutions of generalized Emden-Fowler equations, Trans. Amer. Math. Soc., 167 (1972), pp. 399-434.

[9] L.H. ERBE, Nonoscillation criteria for second order nonlinear differential equations, J. Math. Anal. Appl., 108 (1985), pp. 515-527.

[10] J.W. HeIdeL, A nonoscillation theorem for a nonlinear second order differential equation, Proc. Amer. Math. Soc., 22 (1969), pp. 485-488.

[11] E. Hille, Non-oscillation theorems, Tran. Amer. Math. Soc., 64 (1948), pp. 234-252.

[12] A. KNESER, Untersuchungen über die reelen Nullstellen der Integral linearer Differential gleichungen, Math, Ann., 42 (1893), pp. 409-435; J. Reine Angew. Math., 116 (1896), pp. 178212.

[13] M.K. Kwong, J.S.W. Wong, On an oscillation theorem of Belohorec, SIAM J. Math. Anal., 14 (1983), pp. 474-476.

[14] W.T. LI, R.P. AgARWAL, Oscillation criteria for second order nonlinear differential equations involving integral averages, Comm. Appl. Nonlinear Anal., 8 (2001), pp. 77-87.

[15] M. NAITO, Asymptotic behavior of solutions of second order differential equations with integrable coefficients, Trans. Amer. Math. Soc., 282 (1984), pp. 577-588.

[16] M. NAITO, Integral averages and the asymptotic behavior of solutions of second order ordinary differential equations, J. Math. Anal. Appl., 164 (1992), pp. 370-380.

[17] C.H. Ou, J.S.W. Wong, On existence of oscillatory solutions of second order Emden-Fowler equations, J. Math. Anal. Appl., 277 (2003), pp. 670-680.

[18] C.G. Philos, I.K. Purnaras, On the oscillation of second order nonlinear differential equations, Arch. Math., 59 (1992), pp. 260-271.

[19] J. Sugie ANd T. HARA, Nonlinear oscillations of second order differential equations of Euler type, Proc. Amer. Math. Soc., 124 (1996), pp. 3173-3181.

[20] J. Sugie, K. KitA, Oscillation criteria for second order nonlinear differential equations of Euler type, J. Math. Anal. Appl., 253 (2001), pp. 414-439.

[21] J. Sugie, K. Kita, N. YamaOKa, Oscillation constant of second-order non-linear self-adjoint differential equations, Ann. Mat. Pura Appl., (4) 181 (2002), pp. 309-337.

[22] J. Sugie, N. Yamaoka, An infinite sequence of nonoscillation theorems for second order nonlinear differential equations of Euler type, Nonlinear Anal., 50 (2002), pp. 373-388.

[23] J. Sugie, N. Yamaoka, Oscillation of solutions of second-order nonlinear self-adjoint differential equations, J. Math. Anal. Appl., 291 (2004), pp. 387-405.

[24] J.S.W. Wong, An oscillation theorem for second order sublinear differential equations, Proc. Amer. Math. Soc., 110 (1990), pp. 633-637.

[25] J.S.W. Wong, Oscillation theorems for second-order nonlinear differential equations of Euler type, Methods Appl. Anal., 3 (1996), pp. 476-485. 
